\section{Stochastic Stabilization of Noisy Linear Systems With Fixed-Rate Limited Feedback}

\author{
Serdar Yüksel
}

\begin{abstract}
Fixed-rate quantizers whose bin levels are adaptive have been used in the networked control literature as efficient schemes for stabilizing open-loop unstable noise-free linear systems with arbitrary initial conditions connected over noiseless channels. In this note, stochastic stability results for such simple adaptive quantizers when the system noise has unbounded support for its probability measure are presented. It is shown that, there exists a unique invariant distribution for the state and the quantizer parameters under mild conditions. The second moment under the invariant distribution is finite, if the system noise is Gaussian.
\end{abstract}

Index Terms-Networked control systems, quantization, stability, stochastic systems.

\section{INTRODUCTION}

We consider a remote stabilization problem where a controller having access to quantized measurements acts on a plant, which is open-loop unstable. A setup is depicted in Fig. 1.

Before proceeding further with the description of the system, we discuss the quantization policy investigated. A quantizer, $Q$, for a scalar continuous variable is a map $\mathbb{R} \rightarrow \mathbb{R}$, characterized by a sequence of bins $\left\{\mathcal{B}_{i}\right\}$ and their representation $\left\{q^{i}\right\}$, such that $\forall i, Q(x)=q^{i}$ if and only if $x \in \mathcal{B}_{i}$. Of particular interest is the class of uniform quantizers. In the following, we modify the description of a traditional uniform quantizer by assigning the same value when the state is in the overflow region ${ }^{1}$ of the quantizer. As such, a uniform quantizer: $Q_{K}^{\Delta}: \mathbb{R} \rightarrow \mathbb{R}$ with step size $\Delta$ and $K+1$ (with $K$ even) number of bins satisfies the following for $k=1,2 \ldots, K$ :

$$
Q_{K}^{\Delta}(x)=\left\{\begin{array}{c}
\left(\frac{-(K+1)}{2}+k\right) \Delta, \text { if } x \in\left[\left(\frac{-K}{2}+(k-1)\right) \Delta,\right. \\
\left.\left(\frac{-K}{2}+k\right) \Delta\right) \\
0, \quad \text { if } \quad|x|>\frac{K}{2} \Delta \text { or } x=\frac{K}{2} \Delta .
\end{array}\right.
$$

A general class of quantizers are those which are adaptive. Let $\mathbb{S}$ be a set of states for a quantizer state $S$. Let $F: \mathbb{S} \times \mathbb{R} \rightarrow \mathbb{S}$ be a state update-function. An adaptive quantizer has the following state update equations: $S_{t+1}=F\left(Q_{t}\left(x_{t}\right), S_{t}\right)$. Here, $Q_{t}$ is the quantizer applied at time $t, x_{t}$ is the input to the quantizer $Q_{t}$, and $S_{t}$ is the state of the quantizer. Such a quantizer is implementable since the updates can be performed at both the encoder and the decoder.

One particular class of adaptive quantizers is introduced by Goodman and Gersho [2]. One such type has the following form with $Q_{K}^{\Delta}$ being a uniform quantizer with $K+1$ bins and bin-size $\Delta$ and $\hat{Q}$

Manuscript received April 11, 2009; revised September 09, 2009 and March 15, 2010; accepted June 26, 2010. Date of publication September 27, 2010; date of current version December 02, 2010. This technical note was presented in part at the IEEE International Symposium on Information Theory, 2009 and in part at the IEEE American Control Conference, St. Louis, MO, 2009. This work was supported by the Natural Sciences and Engineering Research Council of Canada (NSERC) in the form of a Discovery Grant. Recommended by Associate Editor Z. Wang.

The author is with the Mathematics and Engineering Program, Department of Mathematics and Statistics, Queen's University, Kingston, ON K7L 3N6, Canada (e-mail: yuksel@mast.queensu.ca).

Digital Object Identifier 10.1109/TAC.2010.2079510

${ }^{1}$ As such, when $|x|>(K / 2) \Delta$, the receiver knows that the source is in the overflow region of the quantizer.

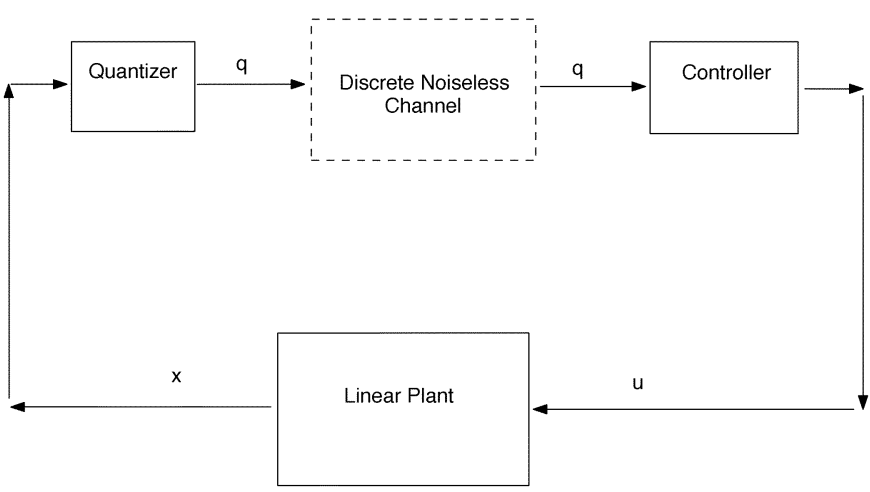

Fig. 1. Control over a finite-rate noiseless channel with fixed-rate quantized observations.

determining the updates in the bin-size of the uniform quantizer as a function of the source and the current bin size:

$$
q_{t}=Q_{K}^{\Delta^{t}}\left(x_{t}\right), \quad \Delta_{t+1}=\Delta_{t} \hat{Q}\left(q_{t}, \Delta_{t}\right) .
$$

Here $\Delta_{t}$ characterizes the uniform quantizer, as it is the bin size of the quantizer at time $t$. In the model that we will consider, $\hat{Q}$ (which we will replace with the term $\bar{Q}$ ) will take ratio between $x$ and $\Delta$, and $\Delta$ as its input.

In this technical note, we consider an LTI discrete-time scalar system described by

$$
x_{t+1}=a x_{t}+b u_{t}+d_{t}, \quad t \in \mathbb{N}
$$

where $a$ is the system coefficient with $|a| \geq 1$, that is, the system is open-loop unstable. We take $b \neq 0$. Here $x_{t}$ is the state at time $t, u_{t}$ is the control input, $x_{0}$ is a second-order random variable, and $\left\{d_{t}\right\}$ is a sequence of zero-mean independent, identically distributed (i.i.d.) random variables which satisfy the following:

Assumption A: $\left\{d_{t}\right\}$ is an i.i.d random sequence such that the random variables admit a probability distribution $\nu$ which is absolutely continuous with respect to the Lebesgue measure on $\mathbb{R}$, and for every open set $D \subset \mathbb{R}, \nu(D)>0$. Furthermore, $E\left[d_{t}^{2}\right]=E\left[d_{1}^{2}\right]<\infty$. $\diamond$

Assumption $B:\left\{d_{t}\right\}$ is an i.i.d. Gaussian sequence with zero-mean and $E\left[d_{1}^{2}\right]<\infty$.

This system is connected over a noiseless channel with a finite capacity to an estimator (controller). The controller has access to the information it has received through the channel. The controller in our model estimates the state and then applies its control. As such, the problem reduces to a state estimation problem since such a scalar system is controllable. Hence, the stability of the estimation error is equivalent to the stability of the state itself.

\section{A. Literature Review and Contributions of the Paper}

There has been considerable amount of research in the literature on quantizer design for such a stabilization problem, for a detailed review see [10]. Due to space constraints, we are unable to provide a detailed account here and only review some of the directly related literature to this note. Zooming type adaptive quantizers, which will be described further in the technical note, have been recently introduced by Brockett and Liberzon [1], for remote stabilization of open-loop unstable, noise-free systems with arbitrary initial conditions. Nair and Evans [4] provided a stability result under the assumption that the quantizer is variable-rate and showed that for a noisy setup (with unbounded support for the noise probability measure) that on average it suffices to use more than $\log _{2}(|a|)$ bits to achieve a form of stability. [4] used 
asymptotic quantization theory to obtain a time-varying scheme, where the quantizer is used at certain intervals at a very high rate, and at other times, the quantizer is not used. Contradistinctively with the result of Nair and Evans, we provide a technique which allows us to both provide a result for the case when the quantizer is fixed-rate as well as to obtain an invariance condition for a probability measure on the quantizer parameters. There is also a large body of literature on quantizer design in the communications and information theory community. One important reference is the work by Goodman and Gersho [2], where an adaptive quantizer was introduced and the adaptive quantizer's stationarity properties were investigated when the source fed to the quantizer is a second order and i.i.d. sequence. In fact, zooming type quantizers is a special class of Goodman and Gersho's adaptive quantization scheme. Kieffer and Dunham [3], have obtained conditions for the stochastic stability of a number of coding schemes when the source considered is also stable, where various forms of stability of the quantizer and the estimation error have been studied. In our case, however, the schemes in [2] and [3] are not directly applicable, as the process we consider is open-loop unstable, as well as Markovian. In view of the literature, the contributions of this note are stated as follows.

- Results on the existence of an invariant distribution is presented. To our knowledge, the first result showing that $\limsup _{t \rightarrow \infty} E\left[x_{t}^{2}\right]=\lim _{t \rightarrow \infty} E\left[x_{t}^{2}\right]<\infty$ is obtained, when the system is driven by a Gaussian disturbance. This result paves the way for solving average cost infinite horizon optimal control problems under quantization constraints.

- The obtained result uses a fixed-rate quantization scheme. This is implementable in a practical setting.

\section{MAIN RESUlts OF THE PAPER}

\section{A. Stochastic Stability of Markov Chains}

Let us first present a brief discussion on stochastic stability of Markov Chains; for a list of definitions on Markov Chains the reader is referred to [5] and [6]. Let $\left\{x_{t}, t \geq 0\right\}$ be a Markov chain with state space $(\mathbb{X}, \mathcal{B}(\mathbb{X}))$, and defined on a probability space $(\Omega, \mathcal{F}, \mathcal{P})$, where $\mathcal{B}(\mathbb{X})$ denotes the Borel $\sigma$-field on $\mathbb{X}, \Omega$ is the sample space, $\mathcal{F}$ a sigma field of subsets of $\Omega$, and $\mathcal{P}$ a probability measure. Let $P(x, D):=P\left(x_{t+1} \in D \mid x_{t}=x\right)$ denote the transition probability from $x$ to $D$.

Definition 2.1: For a Markov chain, a probability measure $\pi$ is invariant on the Borel space $(\mathbb{X}, \mathcal{B}(\mathbb{X}))$ if $\pi(D)=\int_{\mathbb{X}} P(x, D) \pi(d x)$, $\forall D \in \mathcal{B}(\mathbb{X})$.

Definition 2.2: A Markov chain is $\mu$-irreducible, if for any set $B \in$ $\mathcal{B}(\mathbb{X})$ with $\mu(B)>0, \forall x \in \mathbb{X}$, there exists some integer $n>0$, possibly depending on $B$ and $x$, such that $P^{n}(x, B)>0$, where $P^{n}(x, B)$ is the transition probability in $n$ stages, that is $P\left(x_{t+n} \in B \mid x_{t}=x\right)$.

Definition 2.3: A set $A \subset \mathbb{X}$ is $\zeta$-petite on $(\mathbb{X}, \mathcal{B}(\mathbb{X}))$ if for some distribution $\mathcal{T}$ on $\mathbb{N}$ (set of natural numbers), and some non-trivial measure $\zeta, \sum_{n=0}^{\infty} P^{n}(x, B) \mathcal{T}(n) \geq \zeta(B), \forall x \in A, B \in \mathcal{B}(\mathbb{X})$.

Definition 2.4: A $\mu$-irreducible Markov chain is aperiodic if for any $x \in \mathbb{X}$, and any $B \in \mathcal{B}(\mathbb{X})$ satisfying $\mu(B)>0$, there exists $n_{0}=$ $n_{0}(x, B)$ such that $P^{n}(x, B)>0$ for all $n \geq n_{0}$.

Theorem 2.1: ([6] Theorem 4.1) Let $A \in \mathbb{X}$ be a $\zeta$-petite set (for some measure $\zeta$ ). If the Markov Chain is $\mu$-irreducible (for some measure $\mu$ ), aperiodic, and if $\sup _{x \in A} E\left[\min \left(t>0: x_{t} \in A\right) \mid x_{0}=x\right]<$ $\infty$, then the Markov chain is positive Harris recurrent; it thus admits a unique invariant distribution.

The existence of an invariant distribution is important primarily because of the following:

Theorem 2.2 (Birkhoff's Sample Path Ergodic Theorem): Consider a positive Harris recurrent Markov process $\left\{x_{t}\right\}$ taking values in
$\mathbb{X}$, with invariant distribution $\pi($.$) . Let f: \mathbb{X} \rightarrow \mathbb{R}$ be such that $\int f(x) \pi(d x)<\infty$. Then, the following holds almost surely:

$$
\lim _{T \rightarrow \infty} \frac{1}{T} \sum_{t=0}^{T-1} f\left(x_{t}\right)=\int f(x) \pi(d x) .
$$

\section{B. Main Results}

Before proceeding further with the presentation of the main results, we further discuss the adaptive quantizers considered. An example of Goodman-Gersho [2] type adaptive quantizers, which also has been shown to be effective in control systems, are those that have zoom level coefficients as the quantizer state [1]. In the zooming scheme, the quantizer enlarges the bin sizes in the quantizer until the state process is in the range of the quantizer, where the quantizer is in the perfect-zoom phase. Due to the effect of the system noise, occasionally the state will be in the overflow region of the quantizer, leading to an under-zoom phase. We will refer to such quantizers as zooming quantizers. In the following, we will assume the communication channel to be a discrete noiseless one with capacity $R$.

Theorem 2.3: Consider an adaptive quantizer applied to the linear control system described by (2), under Assumption A. If the noiseless channel has capacity, for some $\epsilon>0, R=\log _{2}(\lceil|a|+\epsilon\rceil+1)$, there exists an adaptive quantization policy such that there exists a compact set $S$ with $\sup _{x \in S} E\left[\min \left(t>0: x_{t} \in S\right) \mid x_{0}=x\right]<\infty$, thus $S$ is a recurrent [6] set.

With $K=\lceil|a|+\epsilon\rceil, R=\log _{2}(K+1)$, let us define $R^{\prime}=\log _{2}(K)$. We will consider the following update rules. For $t \geq 0$ and with $\Delta_{0}>$ $L$ for some $L \in \mathbb{R}_{+}$, and $\hat{x}_{0} \in \mathbb{R}$, consider:

$$
u_{t}=-\frac{a}{b} \hat{x}_{t}, \hat{x}_{t}=Q_{K}^{\Delta_{t}}\left(x_{t}\right), \Delta_{t+1}=\Delta_{t} \bar{Q}\left(\left|\frac{x_{t}}{\Delta_{t} 2^{R^{\prime}-1}}\right|, \Delta_{t}\right) \text {. }
$$

If we use $\delta, \epsilon, \eta>0$ with $\eta<\epsilon$ and $L>0$ such that

$$
\begin{aligned}
& \bar{Q}(x, \Delta)=|a|+\delta \quad \text { if } \quad|x|>1 \\
& \bar{Q}(x, \Delta)=\frac{|a|}{|a|+\epsilon-\eta} \quad \text { if } \quad 0 \leq|x| \leq 1, \Delta>L \\
& \bar{Q}(x, \Delta)=1 \quad \text { if } \quad 0 \leq|x| \leq 1, \Delta \leq L
\end{aligned}
$$

with $\left(\sqrt{E\left[d_{t}^{2}\right]} /\left(L(|a| /(|a|+\epsilon-\eta)) 2^{R^{\prime}-1}\right)\right)<\delta$, we will show that a recurrent set exists. We note that the above imply that $\Delta_{t} \geq$ $L(|a| /(|a|+\epsilon-\eta))=: L^{\prime}$ for all $t \geq 0$ (the following are some possible values: $\left.\epsilon=K-|a|, \eta=\epsilon / 2, \delta=\left(E\left[d_{1}^{2}\right]\right)^{1 / 2}\right)$. The proof of Theorem 2.3 is given in Section III-A. Our result on the existence of an invariant distribution is the following, the proof of which is in Section III-B.

Theorem 2.4: Under the setup of Theorem 2.3, for the adaptive quantizer in (3), if the quantizer bin sizes are such that their (base-2) logarithms are integer multiples of some scalar $s$, and $\log _{2}(\bar{Q}(\cdot, \cdot))$ take values in integer multiples of $s$ where the integers taken are relatively prime (that is they share no common divisors except for 1), then the process $\left\{\left(x_{t}, \Delta_{t}\right)\right\}$ is a positive (Harris) recurrent Markov chain, and, as such, has a unique invariant distribution.

Remark 2.1: The existence of an invariant distribution is of fundamental importance in the optimization of infinite horizon stochastic control systems. The Ergodic Theorem lets one adopt the linear programming approach for the optimization of Markov Decision Processes [7].

The following result is on moment stability, the proof of which is presented in Section III-C:

Theorem 2.5: Under the setups of Theorem 2.3, Theorem 2.4 and Assumption B, it follows that $\lim _{t \rightarrow \infty} E\left[x_{t}^{2}\right]<\infty$, and this limit is independent of the initial state of the system. 

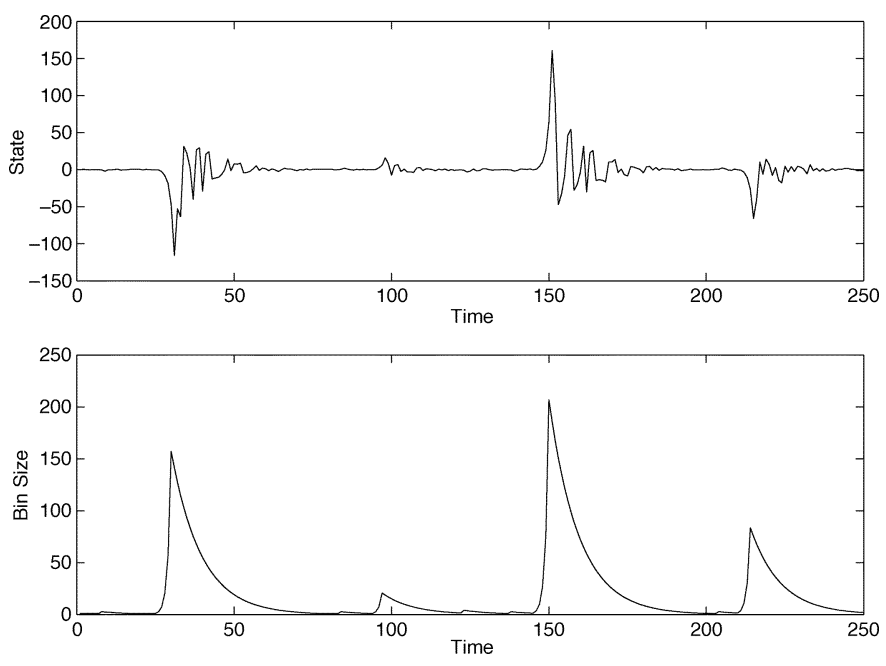

Fig. 2. Sample path for a stochastically stable quantizer. The variables picked are as follows: $L^{\prime}=1, \epsilon=0.3, \delta=0.25, \eta=0.02$.

Remark 2.2: The rate required is close to the lower bound presented by Wong and Brockett: $\log _{2}(|a|)$ [8]. In our case, the additional 1 term is for the overflow bin for the under-zoom phase. As $|a| \rightarrow \infty$, $\log _{2}(|a|+1)-\log _{2}(|a|)=\log _{2}(1+1 /|a|) \leq \log _{2}(e) 1 /|a| \rightarrow 0$, such quantizers are efficient.

Remark 2.3: We note that the stability result for such a scheme requires new techniques to be used, as classical tools in Markovian stability theory will not be applicable directly. This is because of the fact that, the quantizer helps reduce the uncertainty on the system state only when the state is in the granular region of the quantizer. The times when the state is in this region are random. As such, in the following section, we use a two-stage martingale approach to prove the existence of a recurrent set, and subsequently the stability results.

Remark 2.4: We note that, our results above show that there is a limit value for the sequence of second moments $\left\{E\left[x_{t}^{2}\right], t \in \mathbb{Z}_{+}\right\}$. Earlier, Nair and Evans [4] reported that limsup $E\left[x_{t}^{2}\right]<\infty$, using variable-rate schemes. Our result uses a time-invariant rate (that is a fixed-rate) and ensures the existence of a limit distribution.

As a simulation study, we consider a linear system with the following dynamics:

$$
x_{t+1}=2.5 x_{t}+u_{t}+d_{t}
$$

where $E\left[d_{t}\right]=0, E\left[d_{t}^{2}\right]=1$, and $\left\{d_{t}\right\}$ are i.i.d. Gaussian variables. We use the zooming quantizer with rate $\log _{2}(4)=2$, since 4 is the smallest integer as large as $\lceil 2.5\rceil+1$. We have taken $L^{\prime}=1$. Fig. 2 above corroborates the stochastic stability result, by showing the under-zoomed and perfectly-zoomed phases, with the peaks in the plots showing the under-zoom phases.

\section{PROOFS OF THE RESULTS}

\section{A. Proof of Theorem 2.3: Recurrence}

Toward the proof, we will first obtain a supporting result.

Lemma 3.1: Let $\mathcal{B}\left(\mathbb{R} \times \mathbb{R}_{+}\right)$denote the Borel $\sigma$-field on $\mathbb{R} \times \mathbb{R}_{+}$. It follows that:

$$
\begin{aligned}
P\left(\left(x_{t}, \Delta_{t}\right) \in(C \times D) \mid\right. & \left.\left(x_{t-1}, \Delta_{t-1}\right), \ldots,\left(x_{0}, \Delta_{0}\right)\right) \\
& =P\left(\left(x_{t}, \Delta_{t}\right) \in(C \times D) \mid\left(x_{t-1}, \Delta_{t-1}\right)\right)
\end{aligned}
$$

$\forall(C \times D) \in \mathcal{B}\left(\mathbb{R} \times \mathbb{R}_{+}\right)$, i.e. $\left(x_{t}, \Delta_{t}\right)$ is a Markov chain.
Proof: We observe that $x_{t+1}=a x_{t}-a \hat{x}_{t}+d_{t}$, and $\hat{x}_{t+1}=$ $Q_{K}^{\Delta t}\left(x_{t+1}\right)$. Thus

$$
\begin{aligned}
& P\left(\left(x_{t}, \Delta_{t}\right) \in(C \times D) \mid\left(x_{t-1}, \Delta_{t-1}\right), \ldots,\left(x_{0}, \Delta_{0}\right)\right) \\
&= P\left(x_{t} \in C \mid\left(\Delta_{t} \in D, x_{t-1}, \Delta_{t-1}\right), \ldots,\left(x_{0}, \Delta_{0}\right)\right) \\
& \times P\left(\Delta_{t} \in D \mid\left(x_{t-1}, \Delta_{t-1}\right), \ldots,\left(x_{0}, \Delta_{0}\right)\right) \\
&= P\left(x_{t} \in C \mid\left(x_{t-1}, \Delta_{t-1}\right)\right) P\left(\Delta_{t} \in D \mid\left(x_{t-1}, \Delta_{t-1}\right)\right) \\
&= P\left(\left(x_{t}, \Delta_{t}\right) \in(C \times D) \mid\left(x_{t-1}, \Delta_{t-1}\right)\right) .
\end{aligned}
$$

The equations above follow from the update equations in the quantizer (3).

Let us define $h_{t}:=x_{t} /\left(\Delta_{t} 2^{R^{\prime}-1}\right)$. Consider the following sets: $C_{x}=\{x:|x| \leq E\}, C_{h}=\{h:|h| \leq 1\}$, with $E=2^{R^{\prime}-1} L^{\prime}$. Further, let another set be $C_{x}^{\prime}=\{x:|x| \leq F\}$, with a sufficiently large $F$ value to be derived below. We will study the expected number of time stages between visits of $\left\{\left(x_{t}, h_{t}\right)\right\}$ to $C_{x}^{\prime} \times C_{h}$. Consider the drift of the $\left(x_{t}, h_{t}\right)$ process in Fig. 3: When $x_{t}, h_{t}$ are in $C_{x} \times C_{h}$, the expected drift increases both $|h|$ and $|x|$. When the $\left\{x_{t}\right\}$ process gets outside $C_{x}^{\prime}$ and $h_{t}$ outside $C_{h}$ (under-zoomed), there is a drift for $h_{t}$ towards $C_{h}$, however, $\left|x_{t}\right|$ will keep increasing on average. Finally, when the process hits $C_{h}$ (perfect-zoom), then the process drifts towards $C_{x}^{\prime}$. We first show that the sequence $\left\{h_{t}, t \geq 0\right\}$ visits $C_{h}$ infinitely often with probability 1 and the expected length of the excursion is uniformly bounded over all possible values of $(x, h) \in C_{x}^{\prime} \times C_{h}$. Let $V\left(h_{t}\right)=h_{t}^{2}$ serve as a Lyapunov function. Define a sequence of stopping times for the perfect-zoom case with (where the initial state is perfectly zoomed)

$$
\tau_{0}=0, \quad \tau_{z+1}=\inf \left\{k>\tau_{z}:\left|h_{k}\right| \leq 1\right\}, \quad z \in \mathbb{Z}_{+} .
$$

We have that, if $\left|h_{t}\right|>1$ (under-zoomed) $E\left[h_{t+1}^{2} \mid h_{t}, x_{t}\right] \leq\left(\left(a^{2}+\right.\right.$ $\left.\left.\left(E\left[d_{1}^{2}\right] /\left|x_{t}\right|^{2}\right)\right) /(a+\delta)^{2}\right)\left(h_{t}\right)^{2}$. Since when $\left|h_{t}\right|>1$, we have that $\left|x_{t}\right|>2^{R^{\prime}-1} L(|a| /(|a|+\epsilon-\eta))$, it follows that:

$$
E\left[h_{t+1}^{2} \mid h_{t}, x_{t}\right] \leq\left(\frac{a^{2}+\frac{E\left[d_{1}^{2}\right]}{E^{2}}}{(a+\delta)^{2}}\right)\left(h_{t}\right)^{2} .
$$

If $\left|h_{t}\right| \leq 1$, then

$$
\begin{aligned}
E\left[h_{t+1}^{2}\right] & \leq \frac{a^{2} \frac{\left(\Delta_{t}\right)^{2}}{4}+E\left[d_{1}^{2}\right]}{\left(\Delta_{t} 2^{R^{\prime}-1}\right)^{2}}\left(\frac{|a|+\epsilon-\eta}{|a|}\right)^{2} \\
& \leq \frac{a^{2} \frac{L^{\prime 2}}{4}+E\left[d_{1}^{2}\right]}{\left(L^{\prime} 2^{R^{\prime}-1}\right)^{2}}\left(\frac{|a|+\epsilon-\eta}{|a|}\right)^{2}=: K_{1}
\end{aligned}
$$

where $L^{\prime}=L(|a| /(|a|+\epsilon-\eta))$ (this is a lower bound on $\left.\Delta_{t}\right)$. Hence, it follows that:

$$
E\left[h_{t+1}^{2}-h_{t}^{2} \mid h_{t}, x_{t}\right] \leq-\rho h_{t}^{2}+K_{1} 1_{\left\{\left|h_{t}\right| \leq 1\right\}}
$$

where $1_{U}$ is the indicator function for event $U$ with $\rho=1-\left(\left(a^{2}+\right.\right.$ $\left.\left.\left(E\left[d_{1}^{2}\right] / E^{2}\right)\right) /(a+\delta)^{2}\right)$. Since for $A, B>0, A^{2}+B^{2} \leq(A+B)^{2}$ it follows that the hypothesis $\sqrt{E\left[d_{t}^{2}\right]} /\left(L^{\prime} 2^{R^{\prime}-1}\right)<\delta$ ensures $\rho>0$. Now, let us define $K_{1}^{\prime}:=K_{1}+1, M_{0}:=V\left(h_{0}\right)$, and for $t \geq 1$

$$
M_{t}:=V\left(h_{t}\right)-\sum_{i=0}^{t-1}\left(-\rho+K_{1}^{\prime} 1_{\left\{h_{i} \in C_{h}\right\}}\right) .
$$

Define a stopping time: $\tau^{N}=\min \left(N, \min \left\{i>0: V\left(h_{i}\right) \geq\right.\right.$ $\left.N\}, \min \left\{i>0: V\left(h_{i}\right) \leq 1\right\}\right)$. Since, $E\left[M_{t+1} \mid\left(x_{s}, h_{s}\right), s \leq t\right] \leq$ $M_{t}, \forall t \geq 0$, it follows that, $\left\{M_{t}\right\}$ is a supermartingale sequence. The stopping time $\tau^{N}$ is bounded and the supermartingale sequence is also bounded for $t \leq \tau^{N}$. Hence, we have, by the Martingale Optional Sampling Theorem: $E\left[M_{(\tau N)}\right] \leq E\left[M_{0}\right]$. Hence, we obtain 


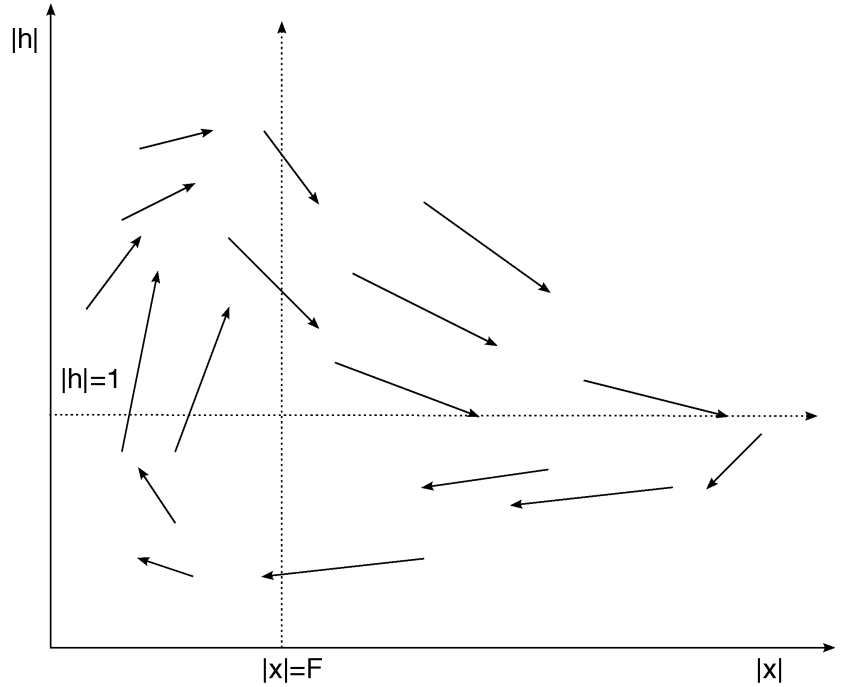

Fig. 3. Drift in the error process. When under-zoomed, the error increases on average; when perfectly-zoomed, the error decreases.

$E\left[\sum_{i=0}^{\tau^{N}-1}\right] \rho \leq V\left(h_{0}\right)+K_{1}^{\prime} E\left[\sum_{i=0}^{\tau^{N}-1} 1_{\left\{h_{i} \in C_{h}\right\}}\right]$. Thus, $\rho E\left[\tau^{N}-\right.$ $1+1] \leq V\left(h_{0}\right)+K_{1}^{\prime}$, and by the Monotone Convergence Theorem

$$
\begin{aligned}
& \rho \lim _{N \rightarrow \infty} E\left[\tau^{N}\right]=\rho E[\tau] \leq V\left(h_{0}\right)+K_{1}^{\prime} \leq 1+K_{1}^{\prime}, \\
& E\left[\tau_{z+1}-\tau_{z}\right] \leq\left(1+K_{1}^{\prime}\right) / \rho
\end{aligned}
$$

uniformly for all $h_{\tau_{z}} \in C_{h}$. By the strong Markov property $\left(x_{\tau_{z}}, h_{\tau_{z}}\right)$ is also a Markov chain as $\left\{\tau_{z}<n\right\} \in \mathcal{F}_{n}$, the filtration generated by the quantizer state and the quantizer output at time $n$. The probability that $\tau_{z+1} \neq \tau_{z}+1$, is upper bounded by the probability

$$
\begin{aligned}
P( & \left\{|a| \Delta_{\tau_{z}} / 2+d_{\tau_{z}} \geq\left(2^{R^{\prime}-1}\right) \Delta_{\tau_{z}} \frac{|a|}{|a|+\epsilon-\eta}\right\} \\
& \left.\bigcup\left\{-|a| \Delta_{\tau_{z}} / 2+d_{\tau_{z}} \leq-\left(2^{R^{\prime}-1}\right) \Delta_{\tau_{z}} \frac{|a|}{|a|+\epsilon-\eta}\right\}\right) \\
= & P\left(\left(d_{\tau_{z}}\right)^{2}>\left((|a| / 2) \Delta_{\tau_{z}} \frac{\lceil|a|+\epsilon\rceil-|a|-\epsilon+\eta}{|a|+\epsilon-\eta}\right)^{2}\right) \\
= & : P_{e}\left(\Delta_{\tau_{z}}\right) .
\end{aligned}
$$

If $\tau_{z+1} \neq \tau_{z}+1$, then this means that the error is increasing on average and the system is once-again under-zoomed at time $t=\tau_{z}+1$ : $x_{\tau_{z}+1}=a x_{\tau_{z}}+d_{\tau_{z}}$ with $\Delta_{\tau_{z}+1}=(|a| /(|a|+\epsilon-\eta)) \Delta_{\tau_{z}}$. (when $\left.\Delta_{\tau_{z}} \geq L\right)$. With some probability, the quantizer will still be in the perfect-zoom phase: $\tau_{z+1}=\tau_{z}+1$. In case perfect-zoom is lost, there is a uniform bound on when the zoom is expected to be recovered. It follows that, conditioned on increment in the error, until the next stopping time, the process will increase exponentially and hence

$$
x_{\tau_{z+1}}=a^{\tau_{z+1}-\tau_{z}}\left(x_{\tau_{z}}+\sum_{t=0}^{\tau_{z+1}-\tau_{z}-1} a^{-t-1} d_{t+\tau_{z}}\right) .
$$

We now show that, there exist $\psi>0,|G|<\infty$ such that

$$
E\left[\log \left(\Delta_{\tau_{z+1}}^{2}\right) \mid \Delta_{\tau_{z}}, h_{\tau_{z}}\right] \leq \log \left(\Delta_{\tau_{z}}^{2}\right)-\psi+G 1_{\left\{\left|\Delta_{\tau_{z}}\right| \leq F\right\}} .
$$

Now, it follows that:

$$
\begin{aligned}
& E\left[\log \left(\Delta_{\tau_{z+1}}^{2}\right) \mid \Delta_{\tau_{z}}, h_{\tau_{z}}\right] \\
& \quad \leq\left(1-P_{e}\left(\Delta_{\tau_{z}}\right)\right)\left(2 \log \left(\frac{|a|}{|a|+\epsilon-\eta}\right)+\log \left(\Delta_{\tau_{z}}^{2}\right)\right) \\
& \quad+P_{e}\left(\Delta_{\tau_{z}}\right) E\left[2\left(\tau_{z+1}-\tau_{z}\right) \log (|a|+\delta)+2 \log \left(\Delta_{\tau_{z}}\right)\right] .
\end{aligned}
$$

We now proceed to further upper bound $E\left[\log \left(\Delta_{\tau_{z+1}}^{2}\right) \mid \Delta_{\tau_{z}}, h_{\tau_{z}}\right]$. Toward this end, we have

$$
\begin{aligned}
& P\left(d_{\tau_{z}}^{2}>\left(\Delta_{\tau_{z}}(|a| / 2)\left(\frac{\lceil|a|+\epsilon\rceil-|a|-\epsilon+\eta}{|a|+\epsilon-\eta}\right)\right)^{2}\right) \\
& \quad \leq \frac{E\left[d_{\tau_{z}}^{2}\right]}{\left(\Delta_{\tau_{z}}(|a| / 2)\left(\frac{\lceil|a|+\epsilon\rceil-|a|-\epsilon+\eta}{|a|+\epsilon-\eta}\right)\right)^{2}} \\
& \quad \leq \frac{E\left[d_{\tau_{z}}^{2}\right]}{\left(K_{2} \Delta_{\tau_{z}}\right)^{2}}
\end{aligned}
$$

where we use Markov's inequality, with $K_{2}=(|a| / 2)((\lceil|a|+$ $\epsilon\rceil-|a|-\epsilon+\eta) /(|a|+\epsilon-\eta))$, and it follows that: $P_{e}\left(\Delta_{\tau_{z}}\right) \leq$ $\left(E\left[d_{\tau_{z}}^{2}\right] /\left(K_{2} \Delta_{\tau_{z}}\right)^{2}\right)$. Now, for large $x_{\tau_{z}}$ values, by substituting the uniform bound in (7), if the following holds, there is a drift to $C_{x}^{\prime}$. For (9) to hold, it suffices that the following equation is satisfied for large enough $\Delta_{\tau_{z}}$ values, for some $\psi>0$ :

$$
\begin{aligned}
P_{e}\left(\Delta_{\tau_{z}}\right)\left\{\left(2\left(1+K_{1}^{\prime}\right) / \rho\right) \log (|a|+\delta)\right\}+ \\
2 \log \left(\frac{|a|}{|a|+\epsilon-\eta}\right) \leq-\psi<0 .
\end{aligned}
$$

Thus, we have $E\left[\log \left(\Delta_{\tau_{z+1}}^{2}\right) \mid \Delta_{\tau_{z}}, h_{\tau_{z}}\right] \leq \log \left(\Delta_{\tau_{z}}^{2}\right)-\psi+$ $G 1_{\left\{\left|\Delta_{\tau_{z}}\right| \leq F^{\prime}\right\}}$ with

$$
F^{\prime}=\frac{\sqrt{E\left[d_{1}^{2}\right]} \sqrt{\left(2\left(1+K_{1}^{\prime}\right) / \rho\right) \log (|a|+\delta)}}{K_{2} \sqrt{-\psi-2 \log \left(\frac{|a|}{|a|+\epsilon-\eta}\right)}}
$$

and $G=2 \log \left(F^{\prime}\right)+2\left(\left(1+K_{1}^{\prime}\right) / \rho\right) \log (|a|+\delta)+\psi$. Hence, we have obtained another drift condition for the sampled Markov chain. This shows that the newly constructed process $\Delta_{\tau_{z}}$ hits the set $\left\{\Delta_{t}\right.$ : $\left.\left|\Delta_{t}\right| \leq F^{\prime}\right\}$ infinitely often. This is equivalent to $x_{\tau_{z}}$ hitting the set $C_{x}^{\prime}=\left\{x:|x| \leq F:=2^{R^{\prime}-1} F^{\prime}\right\}$. Let us call this stopping time process $\tau_{y}^{\prime}$ and define it by $\tau_{0}^{\prime}=\tau_{0}=0$, and for $y>0$

$$
\begin{aligned}
\tau_{y+1}^{\prime} & =\tau_{y}^{\prime}+\inf \left\{t>\tau_{y}^{\prime}:\left|\Delta_{t}\right| \leq F^{\prime},\left|h_{t}\right| \leq 1\right\} \\
& =\tau_{y}^{\prime}+\left(\sum_{l=z+1}^{k} \tau_{l}-\tau_{l-1}\right)
\end{aligned}
$$

with $k=\inf \left\{l>0:\left|\Delta_{\tau_{z+l}}\right| \leq F^{\prime},\left|h_{\tau_{z+l}}\right| \leq 1, \tau_{y}^{\prime}=\tau_{z}\right\}$. Hence, $k$ is the number of visits such that $\left\{h:\left|h_{t}\right| \leq 1\right\}$ until $x_{t}$ hits $C_{x}^{\prime}$. When there is an excursion outside this set, the expected length of the trip (in terms of the original Markov process) is finite, that is $E\left[\tau_{1}^{\prime}\right]<\infty$. This follows because of the following: Define, $M_{0}=\log \left(\Delta_{0}^{2}\right)$, and for some sufficiently small $\alpha>0$

$$
M_{\tau_{z}}=\log \left(\Delta_{\tau_{z}}^{2}\right)-\left(\sum_{k=0}^{\tau_{z}-1}-\alpha+G 1_{\left\{\left|\Delta_{k}\right| \leq F^{\prime}\right\}} 1_{\left\{\left|h_{k}\right| \leq 1\right\}}\right) .
$$

$M_{\tau_{z}}$ is a supermartingale sequence for $0 \leq \tau_{z} \leq \tau_{1}^{\prime}$ if $\alpha<(\psi /((1+$ $\left.\left.K_{1}^{\prime}\right) / \rho\right)$ ), since $E\left[M_{\tau_{z+1}} \mid \mathcal{F}_{\tau_{z}}\right] \leq M_{\tau_{z}}$. For any finite $n$, let us define $k^{n}=\min \left(k, \min \left(l>0: l+\log \left(\Delta_{\tau_{l}}^{2}\right) \geq n\right)\right.$, which is a stopping time. Since $\psi \geq \alpha E\left[\tau_{l+1}-\tau_{l}\right]$, it follows that: $\alpha E\left[\sum_{l=1}^{k^{n}} \tau_{l}-\tau_{l-1}\right] \leq$ $M_{0}+G-2 \log \left(L^{\prime}\right)$. Finally, taking the limit as $n \rightarrow \infty$, and by the Monotone Convergence Theorem, it follows that:

$$
\sup _{\left(x_{\tau_{0}}, h_{\tau_{0}}\right) \in C_{x}^{\prime} \times C_{h}} E\left[\tau_{1}^{\prime}\right] \leq \frac{M_{0}+G-2 \log \left(L^{\prime}\right)}{\alpha} .
$$




\section{B. Proof of Theorem 2.4: Existence and Uniqueness of an Invariant Probability Distribution}

Before proceeding further, let us recall the following.

Lemma 3.2: (Bézout's Lemma, [2]) Let $\{-A, B\}$ be two integers, $A>0, B>0$. Let $\rrbracket$ be the set of all integers that can be obtained by summing positive integer multiples of elements in $\{-A, B\}$. If $A, B$ are relatively prime, then $\rrbracket=\mathbb{Z}$, that is, $\rrbracket$ is the set of all integers.

We now show that the set of admissible quantizers forms a communication class under the hypothesis of the theorem: Since we have $\Delta_{t+1}=\bar{Q}\left(\left|x_{t} /\left(\Delta_{t} 2^{R^{\prime}-1}\right)\right|, \Delta_{t}\right) \Delta_{t}$, it follows that:

$$
\log _{2}\left(\Delta_{t+1}\right) / s=\log _{2}\left(\bar{Q}\left(\left|\frac{x_{t}}{\Delta_{t} 2^{R^{\prime}-1}}\right|\right), \Delta_{t}\right) / s+\log _{2}\left(\Delta_{t}\right) / s
$$

is also an integer. Furthermore, since the source process $x_{t}$ is Lebesgue-irreducible (as the system noise admits a probability density function with positive mass on every open set), and there is a uniform lower bound $L^{\prime}$ on bin-sizes, the error process takes values in any of the admissible quantizer bins with non-zero probability. Let the values taken by $\log _{2}\left(\bar{Q}\left(\left|x_{t} /\left(\Delta_{t} 2^{R^{\prime}-1}\right)\right|, \Delta_{t}\right)\right) / s$ be $\{-A, B\}$, with $A, B$ relatively prime. Thus, for all $l, k \in \mathbb{Z}_{+}, l, k \geq\left(\log _{2}\left(L^{\prime}\right) / s\right)$, there exist $N_{A}, N_{B} \in \mathbb{Z}_{+}$such that $l-k=-N_{A} A+N_{B} B$.

Consider first the case where $k>\left(\log _{2}\left(L^{\prime}\right) / s\right)+N_{A} A$. We show that the probability of $N_{A}$ occurrences of perfect zoom, and $N_{B}$ occurrences of under-zoom phases is bounded away from zero. This set of occurrences includes the event that in the first $N_{A}$ time stages perfect-zoom occurs and later, successively, $N_{B}$ times the under-zoom phase occurs. The probability of this event is lower bounded by

$$
\begin{gathered}
\left(\min _{x:|x| \leq 2 s l_{2} R^{\prime}-1} P\left(d_{t} \in\left[-|a| x-L^{\prime},|a| x+L^{\prime}\right]\right)\right)^{N_{A}} \times \\
\left(\min _{x:|x| \leq 2 s l_{2} R^{\prime}-1} P\left(d_{t}>2^{s l} 2^{R^{\prime}-1}-a x\right)\right)^{N_{B}}>0 .
\end{gathered}
$$

A similar analysis can be performed when $k<\left(\log _{2}\left(L^{\prime}\right) / s\right)+N_{A} A$, by considering the opposite order of events, where in the first $N_{B}$ times, under-zoom occurs, and in the successive $N_{A}$ time stages, perfect-zoom occurs. As such, for any two integers $k, l$ and for some $p>0, P\left(\log _{2}\left(\Delta_{t+p}\right)=l s \mid \log _{2}\left(\Delta_{t}\right)=k s\right)>0$. In our setting, the recurrent set consists of both $\Delta_{t}$ and $e_{t}$. Hence, in this setting, the product space is the product of a discrete-space and a real space. For $x_{t}$, the Borel sets are the open intervals, which are visited infinitely often, and for $\Delta_{t}$, the individual atoms are visited infinitely often with probability 1 .

Now, we can connect these results with Theorems 2.3 and 2.1. We have established irreducibility in the discussion above and recurrence in Theorem 2.3. The chain is aperiodic, as the bin with the smallest admissible size can be visited in subsequent time stages. Finally, the recurrent set $C_{x}^{\prime} \times C_{h}$ is $\kappa$-petite, for some subprobability measure $\kappa$ as any given Borel set is visited starting from the compact set $C_{x}^{\prime} \times C_{h}$ with a uniformly lower bounded probability (over this compact set).

Thus, the chain is positive Harris recurrent.

\section{Proof of Theorem 2.5: Finite Second Moment}

Toward the proof, we state a number of supporting results:

Lemme 3.3: Let $z=0, \tau_{z}=0$ and $\tau_{1}=\tau_{z+1}-\tau_{z}$. It follows that, for $k \in \mathbb{Z}_{+}$:

$$
P\left(\tau_{1}>k\right) \leq C e^{-\left(\left(\zeta^{k-1} N-1 / 2\right) \Delta_{0}\right)^{2} /\left(2 \sigma^{\prime 2}\right)}
$$

with

$$
\begin{aligned}
\sigma^{\prime 2} & =\frac{E\left[d_{1}^{2}\right]}{1-|a|^{-2}}, \zeta=\frac{|a|+\delta}{|a|}, N=\left(2^{R^{\prime}-1} \frac{|a|+\epsilon-\eta}{|a|^{2}}\right), \\
C & =\sigma^{\prime} \frac{2}{\sqrt{2 \pi}\left(\frac{\lceil|a|+\epsilon\rceil}{|a|}-1\right) \Delta_{0} / 2} .
\end{aligned}
$$

Proof: It follows that for $k \geq 1$ :

$$
\begin{aligned}
& P\left(\tau_{1}>k\right) \\
& =P\left(\bigcap _ { t = 1 } ^ { k } \left\{x _ { t } \notin \left[-(|a|+\delta)^{t-1} 2^{R^{\prime}-1} \frac{|a|+\epsilon-\eta}{|a|} \Delta_{0},\right.\right.\right. \\
& \left.\left.\left.2^{R^{\prime}-1}(|a|+\delta)^{t-1} \frac{|a|+\epsilon-\eta}{|a|} \Delta_{0}\right]\right\}\right) \\
& \leq P\left(x _ { k } \notin \left[-(|a|+\delta)^{k-1} 2^{R^{\prime}-1} \frac{|a|+\epsilon-\eta}{|a|} \Delta_{0},\right.\right. \\
& \left.\left.2^{R^{\prime}-1}(|a|+\delta)^{k-1} \frac{|a|+\epsilon-\eta}{|a|} \Delta_{0}\right]\right) \\
& =P\left(a^{k}\left(x_{0}+\sum_{i=0}^{k-1} a^{-i-1} d_{i}\right)\right. \\
& \notin\left[-(|a|+\delta)^{k-1} 2^{R^{\prime}-1} \frac{|a|+\epsilon-\eta}{|a|} \Delta_{0},\right. \\
& \left.\left.2^{R^{\prime}-1}(a+\delta)^{k-1} \frac{|a|+\epsilon-\eta}{|a|} \Delta_{0}\right]\right) \\
& =P\left(\left(x_{0}+\sum_{i=0}^{k-1} a^{-i-1} d_{i}\right)\right. \\
& \notin\left[-\left(\frac{|a|+\delta}{|a|}\right)^{k-1} 2^{R^{\prime}-1} \frac{|a|+\epsilon-\eta}{|a|^{2}} \Delta_{0},\right. \\
& \left.\left.2^{R^{\prime}-1}\left(\frac{|a|+\delta}{|a|}\right)^{k-1} \frac{|a|+\epsilon-\eta}{|a|^{2}} \Delta_{0}\right]\right) \\
& \leq 2 P\left(\sum_{i=0}^{k-1} a^{-i-1} d_{i}>\left(2^{R^{\prime}-1}\left(\frac{|a|+\delta}{|a|}\right)^{k-1}\right.\right. \\
& \left.\left.\times \frac{|a|+\epsilon-\eta}{|a|^{2}}-1 / 2\right) \Delta_{0}\right) \\
& \leq C e^{-\left(\left(\zeta^{k-1} N-1 / 2\right) \Delta_{0}\right)^{2} /\left(2 \sigma^{\prime 2}\right)}
\end{aligned}
$$

with $\sigma^{\prime 2}, \zeta, N$ and $C$ as defined earlier in the Lemma statement. Here, (13) follows from the chain property of a probability measure. Equation (14) follows by bounding the complementary error function: For $u>$ $0: \int_{u}^{\infty} \mu(d x) \leq \int_{u}(x / u) \mu(d x), \mu($.$) being the Gaussian measure. \diamond$

Now, since a decaying exponential decays faster than a polynomial, and the bound above is decreasing in $\Delta_{0}$, there exists an $M<\infty$ such that, with $r>(|a|+\delta)^{2}$, and for all $\Delta_{0} \geq L^{\prime}$

$$
P\left(\tau_{1}=k\right)<C e^{-\left(\left(\zeta^{k-2} N-1 / 2\right) \Delta_{0}\right)^{2} /\left(2 \sigma^{\prime 2}\right)} \leq M r^{-k}, \quad \forall k \in \mathbb{Z} .
$$

Lemma 3.4: We have that, if for some $\gamma>0, b<\infty$, the following holds:

$\gamma E\left[\sum_{k=0}^{\tau_{1}-1} \Delta_{k}^{2} \mid \Delta_{0}, h_{0}\right] \leq \Delta_{0}^{2}-E\left[\Delta_{\tau_{1}}^{2} \mid \Delta_{0}, h_{0}\right]$

$$
+b 1_{\left\{\left(\Delta_{0}, h_{0}\right) \in\left(C_{x}^{\prime} \times C_{h}\right)\right\}}
$$

then, $\lim _{t \rightarrow \infty} E\left[\Delta_{t}^{2}\right]<\infty$ 
The proof of this argument follows from the finite moment drift conditions [6], [9]. Let us now observe that (with a simple bounding argument in the last inequality)

$$
\begin{aligned}
E & {\left[\sum_{t=0}^{\tau_{1}-1} \Delta_{t}^{2} \mid \Delta_{0}\right] } \\
& =\sum_{l=1}^{\infty} P\left(\tau_{1}=l\right) \sum_{k=0}^{l-1} E\left[\Delta_{k}^{2} \mid \tau_{1}=l, \Delta_{0}\right] \\
& \leq \Delta_{0}^{2} \sum_{l=1}^{\infty} P\left(\tau_{1}=l\right) \sum_{k=0}^{l-1}(|a|+\delta)^{2 k} \\
& \leq \Delta_{0}^{2}\left(\sum_{l=1}^{\infty} M\left(r^{-l}\right) \frac{(|a|+\delta)^{2 l}-1}{(|a|+\delta)^{2}-1}\right) \\
& \leq M \Delta_{0}^{2}\left(\frac{1}{1-r^{-1}(|a|+\delta)^{2}}-\frac{1}{1-r^{-1}}\right) \frac{1}{(|a|+\delta)^{2}-1}
\end{aligned}
$$

Let us obtain a bound on $E\left[\Delta_{\tau_{1}}^{2} \mid \Delta_{0}\right]$. It follows that:

$$
\begin{aligned}
E[ & \left.\Delta_{\tau_{1}}^{2} \mid \Delta_{0}\right] \\
= & P\left(\tau_{1}=1\right) E\left[\Delta_{\tau_{1}}^{2} \mid \tau_{1}=1, \Delta_{0}\right] \\
& +P\left(\tau_{1}>1\right) E\left[\Delta_{\tau_{1}}^{2} \mid \tau_{1}>1, \Delta_{0}\right] \\
\leq & P\left(\tau_{1}=1\right) \Delta_{0}^{2}\left(\frac{|a|}{|a|+\epsilon-\eta}\right)^{2} \\
& +P\left(\tau_{1}>1\right) \sum_{k=2}^{\infty} P\left(\tau_{1}=k\right) E\left[\Delta_{k}^{2} \mid \tau_{1}=k, \Delta_{0}\right] \\
\leq & P\left(\tau_{1}=1\right) \Delta_{0}^{2}\left(\frac{|a|}{|a|+\epsilon-\eta}\right)^{2} \\
& +P\left(\tau_{1}>1\right) \sum_{k=2}^{\infty} M r^{-k}(|a|+\delta)^{2 k} \Delta_{0}^{2} \\
\leq & P\left(\tau_{1}=1\right) \Delta_{0}^{2}\left(\frac{|a|}{|a|+\epsilon-\eta}\right)^{2} \\
& +P\left(\tau_{1}>1\right) M\left((|a|+\delta)^{2} r^{-1}\right)^{2} \frac{\Delta_{0}^{2}}{1-r^{-1}(|a|+\delta)^{2}} .
\end{aligned}
$$

Thus, we require, for some $\gamma>0$, and sufficiently large $\Delta_{0}$

$$
\begin{gathered}
\left(P\left(\tau_{1}=1\right) \Delta_{0}^{2}\left(\frac{|a|}{|a|+\epsilon-\eta}\right)^{2}\right. \\
\left.+P\left(\tau_{1}>1\right) M\left((|a|+\delta)^{2} r^{-1}\right)^{2} \frac{\Delta_{0}^{2}}{1-r^{-1}(|a|+\delta)^{2}}\right) \\
<-\gamma M \Delta_{0}^{2}\left(\left(\frac{1}{1-r^{-1}(|a|+\delta)^{2}}-\frac{1}{1-r^{-1}}\right)\right. \\
\left.\quad \times \frac{1}{(|a|+\delta)^{2}-1}\right)+\Delta_{0}^{2} .
\end{gathered}
$$

Since $\lim _{\Delta_{0} \rightarrow \infty} P\left(\tau_{1}>1\right)=0$, by $(10)$

$$
P_{e}\left(\Delta_{\tau_{z}}\right) \leq \frac{E\left[d_{\tau_{z}}^{2}\right]}{\left(K_{2} \Delta_{\tau_{z}}\right)^{2}}
$$

for some sufficiently small $\gamma$, say

$$
\gamma<\frac{\left(1-\left(\frac{|a|}{|a|+\epsilon-\eta}\right)^{2}\right)}{\left(M\left(\frac{1}{1-r-1(|a|+\delta)^{2}}-\frac{1}{1-r-1}\right) \frac{1}{(|a|+\delta)^{2}-1}\right)}
$$

the desired stability result follows for $\left\{\Delta_{t}\right\}$, that is $\lim _{t \rightarrow \infty} E\left[\Delta_{t}^{2}\right]<$ $\infty$.

We have the final result:

Lemma 3.5: If $R>\log _{2}(\lceil|a|+\epsilon\rceil+1)$, then $\lim _{t \rightarrow \infty} E\left[x_{t}^{2}\right]<\infty$.

Proof: Since when $\left|h_{t}\right| \leq 1,\left|x_{t}\right| \leq \Delta_{t} 2^{R^{\prime}-1}$, it suffices to have the following to satisfy $\lim _{t \rightarrow \infty} E\left[x_{t}^{2}\right]<\infty$ : For some $\kappa>0$

$$
\begin{aligned}
\kappa E\left[\sum_{t=0}^{\tau_{1}-1} x_{t}^{2} \mid x_{0}, h_{0}\right] \leq\left(2^{2\left(R^{\prime}-1\right)}\right) \Delta_{0}^{2}- & E\left[x_{\tau_{1}}^{2} \mid x_{0}, h_{0}\right] \\
& +b 1_{\left\{\left(x_{0}, h_{0}\right) \in\left(C_{x}^{\prime} \times C_{h}\right)\right\}} .
\end{aligned}
$$

This is ensured by the finiteness of $E\left[\sum_{t=0}^{\tau_{1}-1} x_{t}^{2} \mid x_{0}, h_{0}\right]$

$$
\begin{aligned}
E & {\left[\sum_{t=0}^{\tau_{1}-1} x_{t}^{2} \mid x_{0}, h_{0}\right]=E\left[\sum_{t=0}^{\infty} 1_{\left\{t<\tau_{1}\right\}} x_{t}^{2} \mid x_{0}, h_{0}\right] } \\
= & E\left[\sum_{t=0}^{\infty} 1_{\left\{t<\tau_{1}\right\}}(|a|+\delta)^{2 t}(|a|+\delta)^{-2 t} x_{t}^{2} \mid x_{0}, h_{0}\right] \\
\leq & \left(E\left[\sum_{t=0}^{\infty} 1_{\left\{t<\tau_{1}\right\}}(|a|+\delta)^{4 t} \mid x_{0}, h_{0}\right]\right)^{\frac{1}{2}} \\
& \times\left(E\left[\sum_{t=0}^{\infty}(|a|+\delta)^{-4 t} x_{t}^{4} \mid x_{0}, h_{0}\right]\right)^{\frac{1}{2}} .
\end{aligned}
$$

In the inequality, we use the Cauchy-Schwarz inequality twice. The first term in (16) is finite by (15), by picking $r$ to be large enough. The second term's boundedness follows:

$$
\begin{aligned}
E & {\left[\sum_{t=0}^{\infty}(|a|+\delta)^{-4 t} x_{t}^{4} \mid x_{0}, h_{0}\right] } \\
& =E\left[\sum_{t=0}^{\infty}\left(\frac{a}{|a|+\delta}\right)^{4 t}\left(x_{0}+\left(\sum_{i=0}^{t-1} a^{-i-1} d_{i}\right)\right)^{4} \mid x_{0}, h_{0}\right] \\
& <\infty .
\end{aligned}
$$

In (17) we use the fact that $\sum_{i=0}^{\infty} a^{-i-1} d_{i}$ is Gaussian with a finite variance of $\sigma^{\prime 2}$, and that the fourth moment of a Gaussian random variable is monotonically increasing in its variance, and that the finite moments of a Gaussian random variable with a finite variance are finite. This completes the proof.

Thus, with $R>\log _{2}(\lceil|a|+\epsilon\rceil+1)$, stability with a finite second moment is achieved. Finally, the limit is independent of the initial distribution since the Chain is irreducible, by Theorem 2.4.

\section{CONCLUSION}

In this technical note, we provided a stochastic stability result for an open-loop unstable system driven by noise using a class of fixed-rate adaptive quantizers. In particular, we showed that such quantizers are rate-efficient, in addition to being simple. One important result in this technical note is the observation that there exists a limit for the sequence of second moments $\left\{E\left[x_{t}^{2}\right], t \in \mathbb{Z}_{+}\right\}$and this limit is finite. This result allows us to formulate an optimal quantization problem when the noise process has unbounded support and the state space for the quantizer bin edges are unbounded. We believe the approach presented in this technical note will find applications in various networked control problems with random stopping times.

\section{REFERENCES}

[1] R. Brockett and D. Liberzon, "Quantized feedback stabilization of linear systems," IEEE Trans. Autom. Control, vol. 45, no. 7, pp. 1279-1289, Jul. 2000. 
[2] D. J. Goodman and A. Gersho, "Theory of an adaptive quantizer," IEEE Trans. Commun., vol. 22, no. 8, pp. 1037-1045, Aug. 1974.

[3] J. C. Kieffer and J. G. Dunham, "On a type of stochastic stability for a class of encoding schemes," IEEE Trans. Inform. Theory, vol. 29, no. 6, pp. 793-797, Nov. 1983.

[4] G. N. Nair and R. J. Evans, "Stabilizability of stochastic linear systems with finite feedback data rates," SIAM J. Control Optim., vol. 43, pp. 413-436, Jul. 2004.

[5] S. P. Meyn and R. Tweedie, Markov Chains and Stochastic Stability. London, U.K.: Springer Verlag, 1993.

[6] S. P. Meyn and R. Tweedie, "Stability of Markovian processes I: Criteria for discrete-time chains," Adv. Appl. Prob., vol. 24, pp. 542-574, 1992.

[7] S. Tatikonda and S. Mitter, "Control under communication constraints," IEEE Trans. Autom. Control, vol. 49, no. 7, pp. 1056-1068, Jul. 2004.

[8] W. S. Wong and R. W. Brockett, "Systems with finite communication bandwidth constraints-Part II: Stabilization with limited information feedback," IEEE Trans. Autom. Control, vol. 42, no. 9, pp. 1294-1299, Sep. 1997.

[9] S. Yüksel, "A random time stochastic drift result and application to stochastic stabilization over noisy channels," in Proc. Allerton Conf., IL, Sep. 2009, pp. 628-635.

[10] S. Yüksel and T. Başar, "Control over noisy forward and reverse channels," IEEE Trans. Autom. Control, to be published.

\section{On Uncontrollable Discrete-Time Bilinear Systems Which are "Nearly" Controllable}

Lin Tie, Kai-Yuan Cai, and Yan Lin

\begin{abstract}
In this note, for a class of uncontrollable discrete-time bilinear systems, it is shown that the controllable region "nearly" covers the whole space while the uncontrollable region is only a hypersurface. As a result, for almost any initial state and any terminal state of the system, the former can be transferred to the latter. In addition, the two-dimensional controllability counterexamples in [1] are generalized to arbitrary finite-dimensional cases.
\end{abstract}

Index Terms-Controllable region, cyclicity, discrete-time bilinear systems, hypersurface, Jacobian determinant, near-controllability, sign patterns.

\section{INTRODUCTION}

Bilinear systems have been investigated for decades owing to their great importance in applications ranging from engineering to economics [1]-[6]. Specifically, the problems of controllability and attainability have been extensively studied in the dynamics and control literature [7]-[16]. For continuous-time bilinear systems, Lie algebra is widely applied to obtain various necessary and sufficient conditions for controllability and attainability [3], [6]. For discrete-time bilinear systems, however, the related results are rather sparse [12]-[16]. Only

Manuscript received April 27, 2009; revised November 05, 2009, March 30, 2010, June 20, 2010; accepted August 24, 2010. Date of publication September 02, 2010; date of current version December 02, 2010. This work was supported by the 973 Program (2010CB327904) and the National Natural Science Foundation of China (60904066, 60874044). Recommended by Associate Editor Z. Wang.

The authors are with the School of Automation Science and Electrical Engineering, Beijing University of Aeronautics and Astronautics, Beijing, 100191, China (e-mail: kingking@asee.buaa.edu.cn; kycai@buaa.edu.cn; linyanee2@yahoo.com.cn).

Digital Object Identifier 10.1109/TAC.2010.2072551 sufficient conditions for controllability were obtained in [12], [13], whereas necessary and sufficient conditions were reported in [15], [16] under strong restrictions on controlled plants. In addition, results on controllability were presented in [17], [18] for analytic and invertible discrete-time nonlinear systems.

In this note, we study the discrete-time homogeneous bilinear system

$$
x(k+1)=(I+v(k) B) x(k)
$$

where $x(k) \in \mathbb{R}^{n}, v(k) \in \mathbb{R}$ and $B \in \mathbb{R}^{n \times n}$. Note that none of the existing results in [12]-[16] is devoted to the controllability of system (1) (although the Lie-algebraic approach presented in [17], [18] is available for investigating the controllability property of such systems, we provide a constructive methodology here). This note will show that, at least under the condition of the eigenvalues of $B$ being non-zero real and distinct, interesting results can be obtained for the controllability problem. Such a matrix can always be put in diagonal form

$$
B=\operatorname{diag}\left(\lambda_{1}, \ldots, \lambda_{n}\right)
$$

by a linear transformation of coordinates. Hence, the system

$$
\begin{aligned}
x(k+1) & =\left(I+v(k)\left[\begin{array}{ccc}
\lambda_{1} & & \\
& \ddots & \\
& & \lambda_{n}
\end{array}\right]\right) x(k) \\
& =\left[\begin{array}{lll}
1+v(k) \lambda_{1} & & \\
& \ddots & \\
& & 1+v(k) \lambda_{n}
\end{array}\right] x(k)
\end{aligned}
$$

is obviously uncontrollable at the state $\xi=\left[\begin{array}{lll}\xi_{1} & \cdots & \xi_{n}\end{array}\right]^{T}$ if some component of $\xi$ vanishes. This may lead one to conjecture that most states of the system (3) are uncontrollable and therefore, (3) is uncontrollable in the "strongest" sense. In this note, we will show the conjecture is, however, invalid. That is, the system (3) has a large controllable region such that for almost any initial state $\xi$ and any terminal state $\eta, \xi$ can be transferred to $\eta$. Accordingly, a new controllability property of discrete-time bilinear systems, i.e., the notion of near-controllability is introduced in this note.

\section{MAIN RESUltS}

Let us first give the following definitions concerning controllability, uncontrollability and near-controllability.

Definition 1 [12], [13]: System (1) is said to be controllable if for any initial state $\xi \in \mathbb{R}_{*}^{n}\left(\mathbb{R}_{*}^{n}:=\mathbb{R}^{n} \backslash\{0\}\right)$ and any terminal state $\eta \in \mathbb{R}_{*}^{n}$, there exist a positive integer $l$ and a finite control sequence $v(k)(k=0,1, \ldots, l)$ such that $\xi$ can be transferred to $\eta$.

Definition 2: A state $\xi$ is said to be uncontrollable if there does not exist an attainable open neighborhood of itself, where an attainable open neighborhood of $\xi$ is an open neighborhood of $\xi$ such that any state belonging to it can be reached from $\xi$.

Definition 3: A system is said to be nearly controllable if there exists a set $A \subset \mathbb{R}^{n}$ of zero Lebesgue measure such that, for any initial state $\xi \in \mathbb{R}^{n} \backslash A$ and any terminal state $\eta \in \mathbb{R}^{n}$, there exist a positive integer $l$ and a finite control sequence $v(k)(k=0,1, \ldots, l)$ such that $\xi$ can be transferred to $\eta$.

Definition 3 is equivalent to that for almost any initial state and any terminal state of the system, the former can be transferred to the latter. Moreover, it implies that almost any state of the system is locally controllable. Next, to prove the main theorem concerning local controllability and near-controllability, we need the following lemmas. 